\title{
Antiferromagnetic and van Hove Scenarios for the Cuprates: Taking the Best of Both Worlds.
}

\author{
Elbio Dagotto, Alexander Nazarenko and Adriana Moreo \\ Department of Physics and National High Magnetic Field Lab, Florida State University, \\ Tallahassee, FL 32306, USA
}

(October 31, 2018)

\begin{abstract}
A theory for the high temperature superconductors is proposed. Holes are spin- $1 / 2$, charge e, quasiparticles strongly dressed by spin fluctuations. Based on their dispersion, it is claimed that the experimentally observed van Hove singularities of the cuprates are likely originated by antiferromagnetic (AF) correlations. From the two carriers problem in the 2D t-J model, an effective Hamiltonian for holes is defined with no free parameters. This effective model has superconductivity in the $\mathrm{d}_{\mathrm{x}^{2}-\mathrm{y}^{2}}$ channel, a critical temperature $\mathrm{T}_{\mathrm{c}} \sim 100 \mathrm{~K}$ at the optimal hole density, $\mathrm{x}=0.15$, and a quasiparticle lifetime linearly dependent with energy. Other experimental results are also quantitatively reproduced by the theory.
\end{abstract}

Typeset using REVTEX 
Recent experimental results for the high temperature cuprate superconductors have suggested that the pairing state is highly anisotropic, probably a $\mathrm{d}_{\mathrm{x}^{2}-\mathrm{y}^{2}}$ singlet. In theories where the pairing mechanism is produced by antiferromagnetic fluctuations, superconductivity in the d-wave channel appears naturally as shown in the context of the 2D Hubbard model using self-consistent techniques, [1] phenomenologically with the nearly-antiferromagnetic Fermi liquid state, [2] and using various techniques in the 2D t-J model. [3] While these approaches seem successful in the prediction of the superconducting symmetry, some phenomenological details of the cuprates remain hidden like the existence of an "optimal" doping. A different family of theories for the cuprates makes extensive use of the concept of van Hove (vH) singularities in the density of states (DOS). [4 In this context, the quasiparticle dispersion is sometimes extracted from angle-resolved photoemission (ARPES) experiments or band structure calculations, a vertex interaction is proposed (usually invoking electronphonon or excitonic mechanisms), and predictions for superconductivity are made using standard techniques. The strong point of this approach is the natural existence of an optimal doping which occurs when the chemical potential reaches the vH singularity.

The purpose of this paper is to describe a microscopically-based theory of the cuprates that combines the strong features of the above described antiferromagnetic and van Hove scenarios. The first step in the construction of such a theory is the observation that the ARPES quasiparticle dispersion may be caused by holes moving in a local antiferromagnetic environment, rather than by band structure effects. This idea is motivated by the existence of universal flat bands [5] near $\mathbf{k}=(\pi, 0),(0, \pi)$ in the spectrum of hole-doped cuprates (2D square lattice language), which are difficult to understand unless caused by correlation effects in the $\mathrm{CuO}_{2}$ planes. Recently, 8,9] it has been shown that models of correlated electrons can account for such flat bands, and here we further elaborate on this idea showing that the agreement with ARPES is quantitative.

Using the well-known two dimensional (2D) t - J model defined by the Hamiltonian,

$$
\mathrm{H}_{\mathrm{tJ}}=-\mathrm{t} \sum_{<\mathrm{i}, \mathrm{j}>\sigma}\left(\mathrm{c}_{\mathrm{i} \sigma}^{+} \mathrm{c}_{\mathrm{j} \sigma}+\text { h.c. }\right)+\mathrm{J} \sum_{<\mathrm{i}, \mathrm{j}>}\left(\mathrm{S}_{\mathrm{i}} \cdot \mathrm{S}_{\mathrm{j}}-\frac{1}{4} \mathrm{n}_{\mathrm{i}} \mathrm{n}_{\mathrm{j}}\right),
$$


in the standard notation, the dispersion of one hole in an antiferromagnet can be calculated accurately [8] with numerical or analytical techniques. At small $\mathrm{J} / \mathrm{t}$, it was found that the hole dispersion is

$$
\epsilon_{\mathbf{k}}=1.33 \mathrm{~J}_{\cos } \mathrm{k}_{\mathrm{x}} \cos \mathrm{k}_{\mathrm{y}}+0.37 \mathrm{~J}\left(\cos 2 \mathrm{k}_{\mathrm{x}}+\cos 2 \mathrm{k}_{\mathrm{y}}\right),
$$

which was calculated using a Green's Function Monte Carlo method. [8] $\mathrm{J}=0.125 \mathrm{eV}$ is the actual scale of the problem, and Eq.(2) shows that holes move within the same sublattice to avoid distorting the AF background. To improve the quantitative agreement with experiments described below, here a small hopping amplitude along the plaquette diagonals $\mathrm{t}^{\prime}=0.05 t$ has been included in the Hamiltonian to produce the dispersion Eq.(2), but the qualitative physics presented in this paper is the same as long as $\left|t^{\prime} / t\right|$ is small. Now, let us assume a rigid band picture for the quasiparticles. [8] The dispersion is plotted in Fig.1a against momentum with the Fermi level at the flat band which corresponds to a hole density of $\mathrm{x}=0.15$ (in Fig.1a and 1c below, $\epsilon_{\mathbf{k}}$ is inverted i.e. the electron language is used rather than the hole language to facilitate the comparison with experiments). $\epsilon_{\mathbf{k}}$ contains a saddle-point located close to $\mathbf{k}=(\pi, 0),(0, \pi)$, which induces a large DOS in the spectrum. In addition, $\epsilon_{\mathbf{k}}$ is nearly degenerate along the $\operatorname{cosk}_{\mathrm{x}}+\cos _{\mathrm{y}}=0$ line increasing the DOS in the vicinity of the Fermi level of Fig.1a. All these qualitative features are common to several models of correlated electrons, and should not be considered as exclusively produced by the t-J model.

What is the influence of a finite hole density on this dispersion? Recent experiments by Aebi et al. [10] have shown the presence of strong antiferromagnetic (AF) correlations in the normal state of BSSCO leading to the formation of hole pockets in their results. A rigid-band filling of $\epsilon_{\mathbf{k}}$ reproduces qualitatively their data (Fig.1b). The most important detail to consider at a small but finite hole density is that the quasiparticle weight is smaller for the bands centered at momentum $(\pi, \pi)$ than those at $(0,0)$ (as represented pictorially in Fig.1a-b, with dashed lines). These are the "shadow bands" which were discussed before in the spin-bag approach. [11] Another argument in favor of using our hole dispersion at a finite 
density also comes from experiments. In Fig.1c we compare $\epsilon_{\mathbf{k}}$ along the $\mathbf{k}=(0,0)-(\pi, 0)$ direction against ARPES results by the Argonne group [5,12] for YBCO. The agreement is excellent. It is worth emphasizing that the theoretical curve of Fig.1c is derived from a microscopic Hamiltonian, and it is not a fit of ARPES data. This is a major difference between the present approach and previous vH scenario calculations.

To further elaborate on whether our dispersion ( derived at $\mathrm{x} \rightarrow 0$ ) can be used at finite $\mathrm{x}$, we have carried out extensive QMC numerical simulations of the 2D Hubbard model at several densities, a large coupling $\mathrm{U} / \mathrm{t}=8$ (i.e. in the regime of the $\mathrm{t}-\mathrm{J}$ model), and temperature $\mathrm{T}=\mathrm{t} / 4$, where $\mathrm{t} \approx 0.4 \mathrm{eV}$. The results (Fig.2a) show that nearest and next-tonearest neighbor spins tend to be antiparallel even with hole densities as large as $\mathrm{x}=0.25$. 13. Reducing the temperature, the correlations would be even stronger than those in Fig.2a. Thus, the assumption that the dispersion Eq.(2) holds near half-filling is supported by numerical and experimental evidence. [14] The intuitive picture to remember is that as long as the antiferromagnetic correlation length is larger than the typical size of a quasiparticle, they will behave as if moving in a nearly perfect AF background.

As a second step in building up a model for the cuprates, the interaction among the hole quasiparticles is necessary, and it will be constructed based again on results obtained for the 2D t-J model. In this case, it is well-known that an effective attractive force exists in an antiferromagnet leading to the bound state of two holes in the d-wave channel. [15] Since this problem is non-trivial, we will simplify its analysis by studying the potential in the atomic limit (large $\mathrm{J} / \mathrm{t}$ ) where the attraction is induced by the minimization of the number of missing antiferromagnetic links [15] (similar to the attraction among spin-bags in a spin-density-wave background [11]). Assuming that the link spin-spin correlation is not much distorted by the carriers in this limit, the binding energy of two holes is $\Delta_{B}=e_{2 h}-2 e_{1 h}=\mathrm{J}\left(\left\langle\mathbf{S}_{\mathbf{i}} \cdot \mathbf{S}_{\mathbf{j}}\right\rangle-0.25\right)$, where $e_{n h}$ is the energy of $n$ holes with respect to the antiferromagnetic ground state energy, and $\mathbf{i}$ and $\mathbf{j}$ are nearest neighbors. Accurate numerical simulations [16] have shown that $\left\langle\mathbf{S}_{\mathbf{i}} \cdot \mathbf{S}_{\mathbf{j}}\right\rangle \approx-0.3346$, and thus $\Delta_{B} \approx-0.6 \mathrm{~J}$. To mimic this effect, an attractive term is introduced that reduces the energy when two quasiparticles share the same link. Thus, the 
Hamiltonian proposed here is

$$
\mathrm{H}=-\sum_{\mathbf{k}, \alpha} \epsilon_{\mathbf{k}} \mathrm{c}_{\mathbf{k} \alpha}^{\dagger} \mathrm{c}_{\mathbf{k} \alpha}-|\mathrm{V}| \sum_{\langle\mathbf{i} \mathbf{j}\rangle} \mathrm{n}_{\mathbf{i}} \mathrm{n}_{\mathbf{j}}
$$

where $c_{\mathbf{k} \alpha}$ is an operator that destroys a quasiparticle with momentum $\mathbf{k}$ and in sublattice $\alpha=\mathrm{A}, \mathrm{B} ; \mathrm{n}_{\mathbf{i}}$ is the number operator at site $\mathbf{i} ;|\mathrm{V}|=0.6 \mathrm{~J}$, and $\epsilon_{\mathbf{k}}$ is given in Eq.(2) [17]. Since in the original t-J language quasiparticles with spin-up(down) move in sublattice $\mathrm{A}(\mathrm{B})$, the interaction term can also be written as a spin-spin interaction. To describe the subspace of zero spin of the original t-J model, half the quasiparticles must be in each sublattice. This Hamiltonian has been deduced based on strong AF correlations, and it has a vH singularity in the noninteracting DOS, thus we will refer to it as the "antiferromagnetic-van Hove" (AFVH) model. Pictorially, it is shown in Fig.2b: quasiparticles move within the A or B sublattices and interact when they share a link. Note that in constructing the low energy AFVH Hamiltonian, retardation effects have been neglected. It will be shown below that this model leads to d-wave superconductivity, which is mainly induced by the real space potential among carriers. [18] Thus, we do not believe that retardation effects will change the conclusions of this paper. The good results described below make this argument selfconsistent.

The AFVH model could be studied using powerful computational techniques, but their implementation is non-trivial. [19] Here, the analysis of the AFVH Hamiltonian will proceed with the standard BCS formalism. Since $|\mathrm{V}| / \mathrm{W} \sim 0.2$, where $\mathrm{W}$ is the bandwidth of the quasiparticles, the gap equation should produce a reliable estimation of the critical temperature since we are effectively exploring the "weak" coupling regime of the AFVH model. Solving the gap equation on $200 \times 200$ grids, we observed that the free energy is minimized using a $\mathrm{d}_{\mathrm{x}^{2}-\mathrm{y}^{2}}$ order parameter. After straightforward algebra it can be shown that the gap equation for the thermodynamical properties in this channel is

$$
\frac{1}{0.6 \mathrm{~J}}=\frac{1}{2 \mathrm{~N}} \sum_{\mathbf{k}} \frac{\operatorname{cosk}_{\mathrm{x}}\left(\operatorname{cosk}_{\mathrm{x}}-\operatorname{cosk}_{\mathrm{y}}\right) \tanh \left(\mathrm{E}_{\mathbf{k}} / 2 \mathrm{~T}\right)}{\mathrm{E}_{\mathbf{k}}} \text {, }
$$

where $\mathrm{E}_{\mathbf{k}}=\sqrt{\left(\epsilon_{\mathrm{k}}-\mu\right)^{2}+\Delta_{\mathrm{k}}^{2}}, \Delta_{\mathrm{k}}=\frac{\Delta_{0}}{2}\left(\operatorname{cosk}_{\mathrm{x}}-\operatorname{cosk}_{\mathrm{y}}\right), \Delta_{0}$ is the parameter to be obtained 
self-consistently, $\mathrm{N}$ is the number of sites, and $\mathrm{T}$ is the temperature. Note that the $\mathrm{T}$ dependence of $\mu$ cannot be neglected. In Fig.2c, $T_{c}$ against the hole density is shown. 20] Two features need to be remarked: i) an optimal doping exists at which $\mathrm{T}_{\mathrm{c}}$ is maximized which is a direct consequence of the presence of a large peak in the DOS of the quasiparticles; ii) the optimal doping $(15 \%)$ and optimal $\mathrm{T}_{\mathrm{c}}$ of about $100 \mathrm{~K}$ are in excellent agreement with the cuprates phenomenology. 21] Although in the effective AFVH Hamiltonian the natural scale of the problem is $\mathrm{J} \sim 1000 \mathrm{~K}$, since the ratio between coupling and bandwidth is small, $\mathrm{T}_{\mathrm{c}}$ is further reduced in the weak coupling BCS formalism to about $100 \mathrm{~K}$. Note that this quantitative agreement with experiments is obtained without the need of ad-hoc fitting parameters. It is also important to remark that the presence of a finite optimal density of holes cannot be achieved if a simpler $\left(\cos k_{x}+\cos k_{y}\right)^{2}$ dispersion is used. Thus, as noticed in Ref. [8], the small energy difference between $\mathbf{k}=(\pi / 2, \pi / 2)$ and $(\pi, 0)$ in the hole dispersion is crucial for the quantitative success of this approach.

From the gap equation, the ratio $\mathrm{R}(\mathrm{T})=2 \Delta_{\max }(\mathrm{T}) / \mathrm{kT}_{\mathrm{c}}$ can be calculated, (for a d-wave condensate at temperature $\mathrm{T}, \Delta_{\max }(\mathrm{T})$ is defined as the maximum value of the gap). In Fig.3a, $\mathrm{R}(\mathrm{T})$ is shown as a function of temperature, compared with recent tunneling data. [22] At $\mathrm{T}=0$, the AFVH model predicts $\mathrm{R}(0)=5.2$ and the tunneling experiment gives 6.2. While the agreement is already encouraging, note that other experiments have reported a smaller value for $\mathrm{R}(0)$. For example, ARPES data by Ma et al. [7] obtained $\mathrm{R}(0)=4.6$, while an average over the pre-1992 literature [23] suggested $\mathrm{R}(0)=5 \pm 1$ supporting the results of the AFVH model. At $T_{c}$ we have also calculated the ratio $R_{2}=\Delta C / C_{n}$, where $\Delta \mathrm{C}=\mathrm{C}_{\mathrm{s}}-\mathrm{C}_{\mathrm{n}}$ is the difference between the specific heat of the normal state $\mathrm{C}_{\mathrm{n}}$ (calculated by turning off the interaction $|\mathrm{V}|$ ), and the superconducting state $\mathrm{C}_{\mathrm{s}}$. The result is shown in Fig.3b. At $\mathrm{T}_{\mathrm{c}}, \mathrm{R}_{2} \approx 4.2$ which is again in good agreement with $\mathrm{YBCO}$ experiments reported by Phillips et al., and Loram and Mirza [24] which found $\mathrm{R}_{2}=4.8$ and 4.1, respectively. It is also gratifying that not only dimensionless ratios but also absolute values are in reasonable agreement with experiments. For example, in Fig.4a $\Delta \mathrm{C} / \mathrm{T}_{\mathrm{c}}$ is shown as a function of density compared with results for YBCO. Although the relation between hole carriers and 
the amount of oxygen in this material is unknown, the general trends between theory and experiment are similar, as was observed in other vH calculations. 255]

We have also verified that an important feature of previous $\mathrm{vH}$ scenarios also exists in our model, i.e. a quasiparticle lifetime linear with frequency at the optimal doping. To carry out the calculation we switched off the attractive term, introduce particle-particle repulsion within the same sublattice at distance one, [27] and carry out the standard one bubble approximation to get the imaginary part of the self-energy. The result shows a linear behavior at the optimal doping (Fig.4b). Finally, let us address the stability of the van Hove singularity against effects not explicitly considered in the Hamiltonian. In some van Hove theories the singularity in the DOS is rapidly removed by inhomogeneities or 3D effects. For example, the DOS of a $2 \mathrm{D}$ tight-binding model with dispersion $\epsilon_{\mathbf{k}}=-2 \mathrm{t}\left(\operatorname{cosk}_{\mathrm{x}}+\operatorname{cosk}_{\mathrm{y}}\right)$ is plotted in Fig.4c with an energy-independent broadening $\Gamma=0.02 \mathrm{eV}$ using $\mathrm{N}(\omega)=\frac{2}{\pi \mathrm{N}} \sum_{\mathbf{k}} \frac{\Gamma}{\left(\omega-\epsilon_{\mathbf{k}}\right)^{2}+\Gamma^{2}}$, and $\mathrm{t}=0.4 \mathrm{eV}$. Fig.4c shows that the singularity is unobservable with this broadening. However, for the dispersion of the AFVH model the situation is different since the carriers bandwidth is narrow, with a scale setup by $\mathrm{J}$, and there is a large accumulation of weight in the DOS at low energy. In other words, the number of states in the vicinity of the $\mathrm{vH}$ singularity is of the order of the total number of states in the full band. For these reasons the influence of disorder on the DOS is milder in the AFVH model, where a large peak can still be seen in Fig.4c even with a broadening $\Gamma=0.02 \mathrm{eV}$.

In this paper, simple and quantitative ideas for high-Tc superconductivity have been discussed combining for the first time two apparently different proposals for the cuprates i.e. the antiferromagnetic and van Hove scenarios. We have claimed that these approaches are actually deeply related with both the pairing mechanism and the $\mathrm{vH}$ singularity in the DOS of the high Tc materials caused by antiferromagnetic correlations. This theory explains in an economical way the $d_{x^{2}-y^{2}}$ superconducting state apparently observed in several experiments, and goes beyond previous work showing that the critical temperature is maximized at a particular optimal doping where the quasiparticle lifetime is linear with energy. An excellent quantitative agreement between theory and experiments is reported 
for the critical temperature, optimal doping, superconducting gap and specific heat.

We thank D. S. Dessau, K. Gofron, D. W. Hess, M. Horbach, J. Kim, D. M. King, J. Ma, R. S. Markiewicz, M. Onellion, J. Osterwalder, J. Riera, J. R. Schrieffer, Z.-X. Shen, and B. O. Wells, for useful conversations and suggestions. E. D. and A. M. are supported by the Office of Naval Research under grant ONR N00014-93-0495. Part of this work was supported by the National High Magnetic Field Laboratory, Tallahassee, FL. 


\section{REFERENCES}

[1] N. E. Bickers, D. J. Scalapino, and S. R. White, Phys. Rev. Lett. 62, 961 (1989).

[2] P. Monthoux and D. Pines, Phys. Rev. Lett. 69, 961 (1992).

[3] E. Dagotto and J. Riera, Phys. Rev. Lett. 70, 682 (1993); E. Dagotto, et al., Phys. Rev. B 49, 3548 (1994); Y. Ohta et al., preprint; E. Heeb and T. M. Rice, preprint.

[4] C. C. Tsuei et al., Phys. Rev. Lett. 65, 2724 (1990); R. S. Markiewicz, J. Phys. Condens. Matt. 2, 6223 (1990); D. M. Newns, P. C. Pattnaik and C. C. Tsuei, Phys. Rev. B 43, 3075 (1991); R. S. Markiewicz, Int. J. Mod. Phys. B5, 2037 (1991); and references therein.

[5] K. Gofron et al., J. Phys. Chem. Solids 54, 1193 (1993).

[6] D. S. Dessau et al., Phys. Rev. Lett. 71, 2781 (1993); D. M. King et al., preprint; B. O. Wells et al., preprint.

[7] Jian Ma et al., Madison preprint, May 1994.

[8] E. Dagotto, A. Nazarenko and M. Boninsegni, to appear in Phys. Rev. Letters.

[9] N. Bulut, D. J. Scalapino and S. R. White, preprint.

[10] P. Aebi et al., Phys. Rev. Lett. 72, 2757 (1994).

[11] A. Kampf, and J. R. Schrieffer, Phys. Rev. B42, 7967 (1990), and references therein.

[12] A. A. Abrikosov, J. C. Campuzano, and K. Gofron, Physica C 214, 73 (1993).

[13] A. Moreo and D. Duffy, preprint (in preparation).

[14] S. Trugman, Phys. Rev. Lett. 65, 500 (1990) also proposed a rigid-band approach to hole quasiparticles.

[15] For references see E. Dagotto, Rev.Mod.Phys., July 1994. 
[16] J. Carlson, Phys. Rev. B40, 846 (1989).

[17] In other words, the quasiparticle interaction is short-ranged and attractive (see e.g. D. Poilblanc et al., Phys. Rev. B 49, 12318 (1994); M. Yu. Kuchiev and O. P. Sushkov, Physica C 218, 197 (1993)), thus the Hamiltonian Eq.(3) is natural. A similar approach in the t-J model was followed by J. Inoue and S. Maekawa, Prog. of Theor. Phys. 108, 313 (1992).

[18] D. J. Scalapino et al., preprint.

[19] J. Riera et al., work in progress.

[20] We checked that Hartree-Fock corrections are small, and do not alter the features of the dispersion Eq.(2).

[21] A similar Tc was found by C-H. Pao and N. E. Bickers, Phys. Rev. Lett. 72, 1870 (1994); and P. Monthoux and D. Scalapino, Phys. Rev. Lett. 72, 1874 (1994).

[22] S. I. Vedeneev et al., Phys. Rev. B 49, 9823 (1994).

[23] B. Batlogg, Springer Series in Solid-State-Sciences, Vol. 106, Eds.: S. Maekawa and M. Sato, page 219 (1992).

[24] N. Phillips et al., Phys. Rev. Lett. 65, 357 (1990); J. W. Loram and K. A. Mirza, Physica C 153, 1020 (1988).

[25] C. C. Tsuei et al., Phys. Rev. Lett. 69, 2134 (1992).

[26] M. Däumling, Physica C183, 293 (1991).

[27] This interaction does not alter the d-wave condensate, and it stabilizes it against phase separation effects.

\section{Figure Captions}

1. (a) Quasiparticle dispersion of the 2D t-J model (Eq.(2)) using $\mathrm{J}=0.125 \mathrm{eV}$. The 
electron notation is used, thus all levels below $\mathrm{E}_{\mathrm{F}}$ are occupied. Thick lines are the position of the quasiparticles that should be easily observed in ARPES experiments at a finite hole concentration. The dashed line represents the "shadow dispersion", caused by the remnant $\mathrm{AF}$ at finite density. The calculation was done with a Monte Carlo technique (Ref. [8]); (b) Experimental results for Bi2212 (Ref. [10]) The $\bar{M}(Y)$ point corresponds to $\mathbf{k}=(\pi, 0)((\pi, \pi))$ in the 2D square lattice language. The thick line is a strong experimental signal, while the dashed line is weak; (c) Direct comparison between experiments and theory: the solid line is our dispersion Eq.(2), while the experimental results are from Ref. [12].

2. (a) Spin-spin correlation $C(\mathbf{r})=\left\langle S_{\mathbf{i}}^{z} S_{\mathbf{i}+\mathbf{r}}^{z}\right\rangle(-1)^{|\mathbf{r}|}$ vs distance for the $2 \mathrm{D}$ Hubbard model calculated using Quantum Monte Carlo at $\mathrm{U} / \mathrm{t}=8$ on an $8 \times 8$ cluster. The electronic densities starting from above are $1.0,0.90,0.83$, and 0.74 . The temperature is $\mathrm{T}=0.1 \mathrm{eV}, \Delta \tau=0.0625$, and the error bars are typically about 0.02 ; (b) a pictorial representation of the AFVH model (see text); (c) Critical temperature $\mathrm{T}_{\mathrm{c}}$ of the AFVH model as a function of hole density $\mathrm{x}(=1-\langle n\rangle)$. The superconducting state is d-wave.

3. (a) $2 \Delta_{\max }(T) / \mathrm{kT}_{\mathrm{c}}$ against $\mathrm{T} / \mathrm{T}_{\mathrm{c}}$. The solid line corresponds to the AFVH model at the optimal doping. The open squares are tunneling data for $\mathrm{Bi}_{2} \mathrm{Sr}_{2} \mathrm{CaCu}_{2} \mathrm{O}_{8}[22]$. The full triangle at $\mathrm{T}=0$ corresponds to ARPES data [7] while the full square is a summary of experimental data. 23] The dotted line is the BCS prediction (i.e. attractive Hubbard model at half-filling and weak coupling); (b) $R_{2}$ as defined in the text vs $T / T_{c}$ for the AFVH model at the optimal doping. The dot corresponds to experimental results for YBCO. [24]. The BCS result is shown.

4. (a) $\Delta \mathrm{C} / \mathrm{T}_{\mathrm{c}}$ vs hole density $\mathrm{x}$ using the AFVH model. Open squares correspond to experimental data [26] for $\mathrm{YBa}_{2} \mathrm{Cu}_{3} \mathrm{O}_{7-\delta}$, using the convention that the result at the optimal $\delta(\sim 0.02)$ is plotted at our optimal doping $\mathrm{x} \sim 0.15$, and that 
$\delta \approx 0.02+(0.15-\mathrm{x})$ in the rest of the data. The vertical axis is in $\mathrm{mJ} / \mathrm{K}^{2} / \mathrm{mol}$ units for the experiment and $\mathrm{mJ} / \mathrm{K}^{2} /$ site for the theory; (b) Quasiparticle lifetime vs. energy, $\omega$, at $\mathrm{T}=0$. The solid line corresponds to the chemical potential, $\mu$, at the saddle-point, while for the dashed line $\mu$ is above the saddle-point. Vertical units are arbitrary; (c) DOS corresponding to the AVFH model (I), and a tight-binding model (II), using $\Gamma=0.02 \mathrm{eV}$. 